\title{
Dental caries among Finnish teenagers participating in physical activity and diet intervention: association with anthropometrics and behavioural factors
}

Mirja Methuen ${ }^{1,2,3^{*}{ }^{\dagger}}$, Sofia Kauppinen ${ }^{1 \dagger}$, Anna Liisa Suominen ${ }^{1,2}$, Aino-Maija Eloranta ${ }^{4,5}$, Juuso Väistö ${ }^{5}$, Timo Lakka ${ }^{5,6,7}$, Hannu Vähänikkilä ${ }^{8}$ and Vuokko Anttonen ${ }^{3,9}$

\begin{abstract}
Background: An association between childhood anthropometric measurements and dental caries is conflicting. The prevalence and severity of dental caries and its association with anthropometric and behavioural factors, were investigated among Finnish teenagers.
\end{abstract}

Methods: The study sample comprised 202 15-17-year-old participants in the Physical Activity and Nutrition in Children (PANIC) Study. Dental caries findings were recorded using International Caries Detection and Assessment System (ICDAS) criteria, including activity estimation; numbers of decayed teeth (DT) and decayed, missing and filled teeth (DMFT) were recorded. Body weight, height and waist circumference were measured and respective body mass index (BMI) was calculated. Body fat percentage was assessed by dual-energy X-ray absorptiometry. Health-related behaviours and consumption of food and drinks were assessed using questionnaires, and intake of nutrients using a 4-day food record.

Results: Mean DMFT for all the participants was $2.4(\mathrm{SD}=2.9)$, DT $0.6(\mathrm{SD}=1.3)$, and $36 \%$ had DMFT = 0 . No difference between genders was observed. In bivariate analyses, use of sugar-sweetened beverages (SSB) three times or less per week and not having used snuff associated significantly, whereas higher carbohydrate intake (E\%), toothbrushing less often than twice a day and higher caries experience at baseline almost significantly with DT $>0$. In adjusted regression analyses, frequent use of SSB and higher carbohydrate intake increased the odds for DT $>0$. Additionally, higher carbohydrate intake (E\%) and infrequent tooth brushing significantly associated with a higher number of DT.

Conclusion: Caries prevalence is still low and similar in Finnish teenage girls and boys. Behavioural factors are, but anthropometric factors are not associated with dental caries.

Keywords: Adolescent, Body mass index, Dental caries, Diet, Oral health behaviour

*Correspondence: mirja.methuen@uef.fi

${ }^{\dagger}$ Mirja Methuen and Sofia Kauppinen have contributed equally to this work

${ }^{1}$ Institute of Dentistry, Faculty of Health Sciences, University of Eastern Finland, P.O. Box 1627, 70211 Kuopio, Finland

Full list of author information is available at the end of the article

\section{Background}

In 2017, oral diseases affected approximately $47 \%$ of the world's population. About 31\% had untreated caries in permanent teeth and half a billion in primary dentition [1]. World population in 2017 was 7.5 billion and in 2020 it was 7.8 billion [2]. Caries exists everywhere, although 
untreated caries is more prevalent in the developing world. In Finland, the mean number of decayed, missing and filled teeth (DMFT) for 12-year-olds fell from 6.9 to 1.2 between 1975 and 1994, and a similar trend was seen in the other age groups of children and adolescents [3]. Since then, a plateauing in the decline of caries has been seen $[4,5]$. WHO school oral health surveys provide data on health behaviour every second year, but since the end of the 1990s there is no evidence-based data available on the prevalence of dental caries among Finnish children and adolescents.

Anthropometry refers to "the measurement of the size, weight, and proportions of the human or other primate body" [6]. Anthropometric measurements such as height and body mass index (BMI) are important indicators of children's growth and development and they are correlated with children's nutritional status [7]. Children's overweight and obesity are common in today's industrialized world, even though the increasing trend has somewhat plateaued [8]. Overweight or obesity have increased in 12-18-year-old Finnish adolescents up to two-threefold between 1997 and 2003 [9]. In 2018, nearly 25\% of Finnish children and adolescents were overweight, boys more often than girls [10]. Caries and obesity are both multifactorial diseases sharing similar risk factors such as a harmful diet, socioeconomic conditions [11] and lifestyle [12]. Literature on the association between overweight and caries is contradictory; both high and low BMI showing an association with decaying teeth in children $[13,14]$. The association depends on the country's stage of development as well as on socioeconomics [13]. In Hooley's et al. [13] systematic review, a positive association was found primarily in highly developed countries such as in Europe and the United States, whereas an inverse association or association of higher number of decayed teeth with lower BMI was found in Asia and South America. Further, Li et al. [15] in their systematic review concluded that the evidence for an association between childhood anthropometric measurements and dental caries is still conflicting and remains inconclusive.

Dietary habits have changed over the past decades. Snacking has replaced meals and beverages are consumed more frequently than ever [16, 17]. Frequent snacking and intake of sweet products are associated with overweight [18] as well as with dental caries [19]. Snacking is also associated with other harmful oral and general health habits such as smoking, snuffing and infrequent tooth brushing [20]. The difference between the genders in caries prevalence of Finnish school children appears to be disappearing [21], but girls still have slightly better oral health behaviours than boys in terms of use of sugarsweetened beverages (SSB) and tooth brushing frequency $[22,23]$.
This paper aims to bring new information on caries experience and its distribution between genders. Physical Activity and Nutrition in Children (PANIC) study cohort gives an opportunity to study anthropometrics and behavioural factors in association with dental caries. The hypotheses were that boys have more dental caries than girls, and anthropometrics and high sugar intake as well as infrequent tooth brushing are associated with a higher prevalence of dental caries.

\section{Methods}

\section{Study design and study population}

The present analyses are based on data from the Physical Activity and Nutrition in Children (PANIC) study, which is a controlled physical activity and dietary intervention study with an ongoing follow-up in a population sample of children from the city of Kuopio, Finland. Altogether 736 children of 6-8 years of age who started the first grade in primary schools in Kuopio between 2007 and 2009 were invited to participate in the study. The participants were divided into intervention and control groups. The intervention included physical activity and dietary counselling sessions during the follow-up (0.5, $1.5,3,6,12,18,24,36,48,60,72,84$, and 96 months after baseline) [24]. The children and their parents in the control group received general verbal and written advice on health improving physical activity and diet at baseline but no active intervention. The protocol of the study is described in Fig. 1. At baseline and at 2-year follow-up, dental examination was done as suggested by the WHO. Dental examination at 8-year follow-up is described in the next chapter. Public health dentists recorded teeth as having initial lesions (I), being decayed (D), missing (M) or/and having fillings (F). This protocol has been found to be reliable [25].

Data for the present analyses were obtained from the 8-year follow-up study of 202 children between 2016 and 2018 . The study group consisted of $48 \%$ girls and $52 \%$ boys, the mean age (SD) being $16.5(0.5)$ years.

\section{Assessment of dental caries and need for restorative treatment}

At 8-year follow up, participants were called to a separate appointment for the dental examination. Dental examinations were carried out in a dental office at the University of Eastern Finland by an experienced dentist (MM), specialist in cariology and endodontics. MM performed all the examinations except five, which were done by a senior researcher VA with the same field of expertise. The research dentist (MM) was trained and calibrated for examinations by VA familiar with such trainings (VA) $[26,27]$. Training on the study protocol was organised and criteria were reviewed using a PowerPoint 

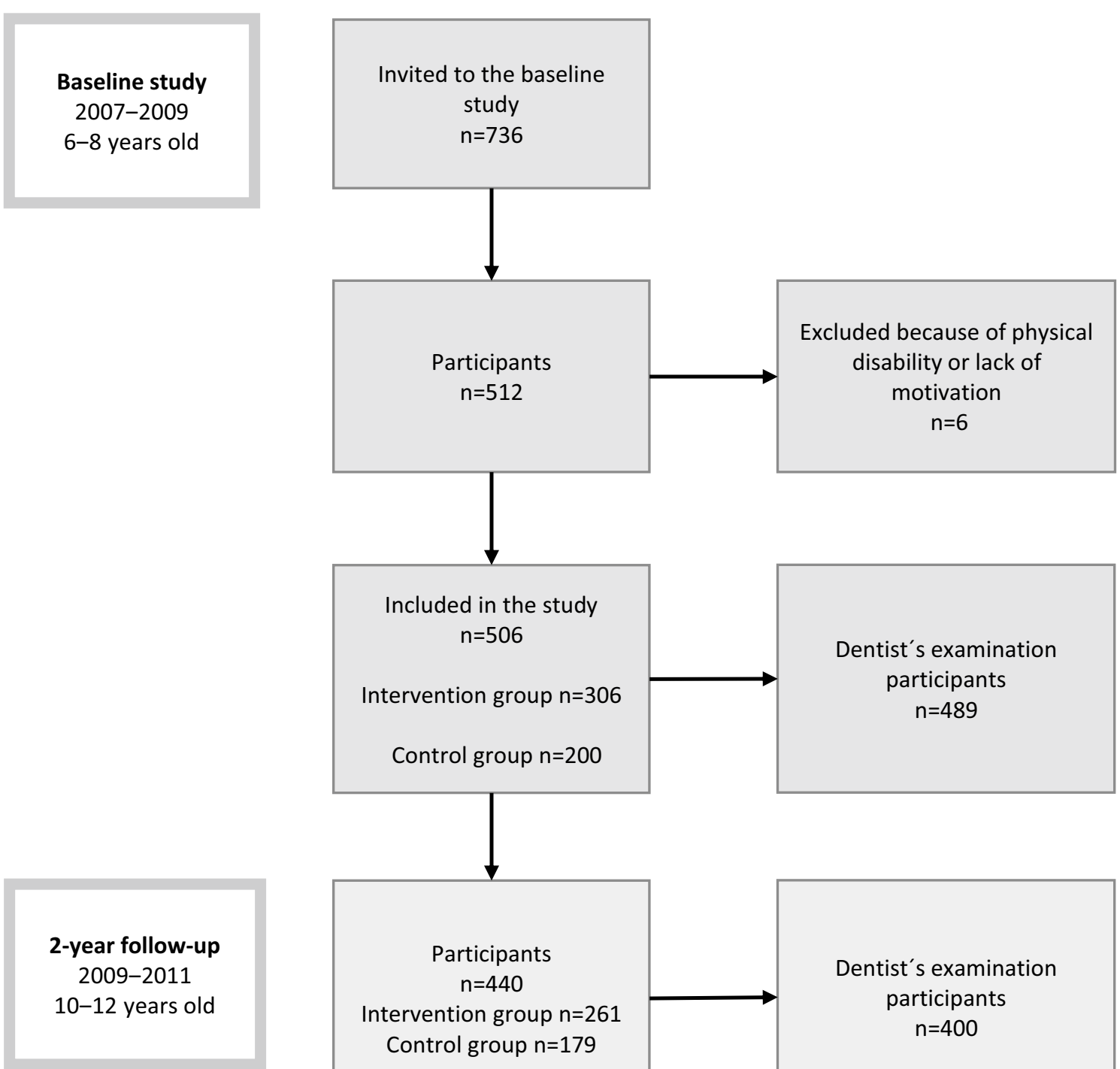

Included in the study $n=506$

Intervention group $\mathrm{n}=306$

Control group $n=200$

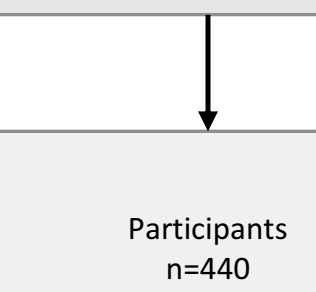

Intervention group $n=261$

Control group $n=179$
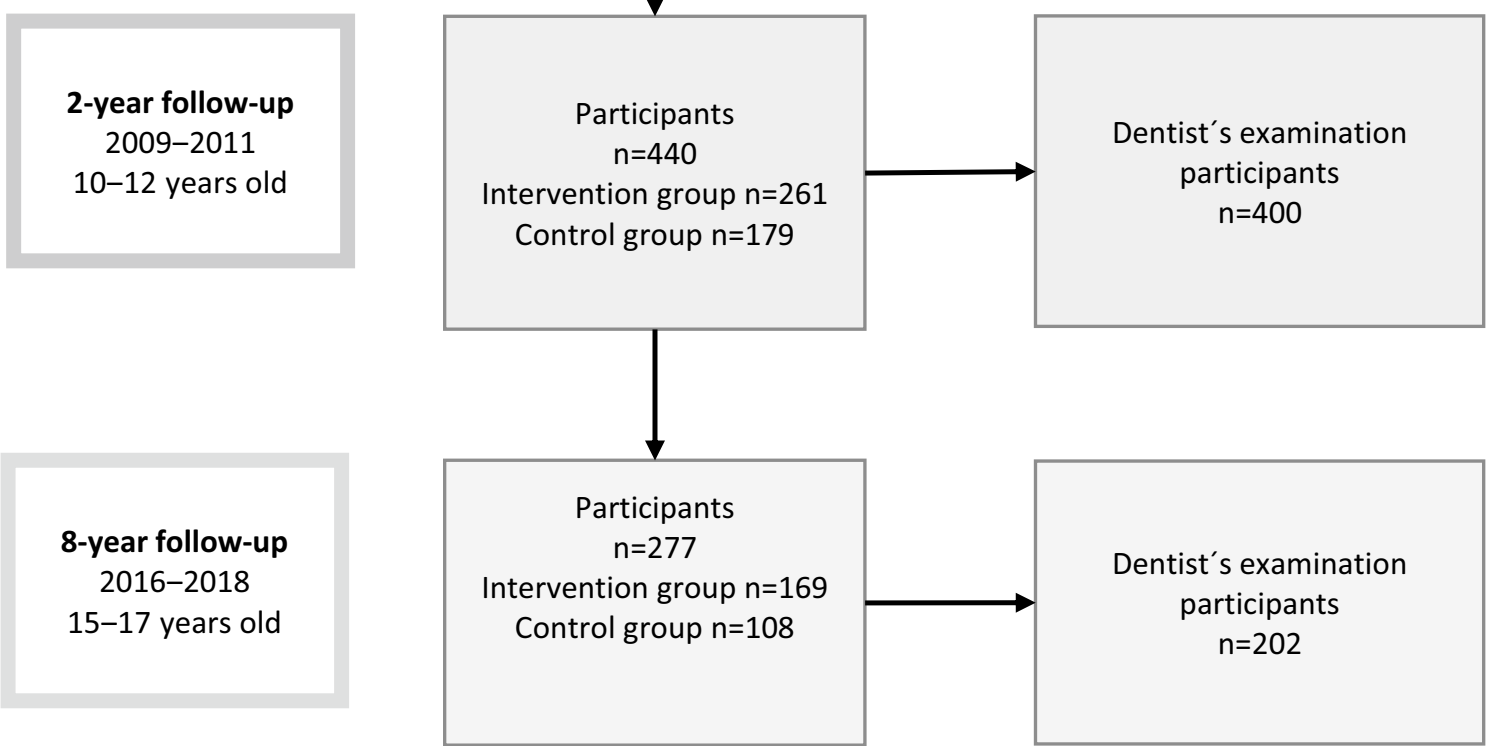

Fig. 1 A flow-chart presenting the participants at baseline, at 2-year and 8-year follow-ups of the Physical Activity and Nutrition in Children (PANIC) study

presentation. Images of extracted teeth were used to demonstrate different stages of dental caries lesions according to the International Caries Detection and
Assessment System (ICDAS) [28]. During the training session the senior researcher monitored MM's work for two days (6 patients) to calibrate and verify the protocol 
of the clinical examination. Training and calibration were repeated every three months. To investigate the reliability of the examinations, parallel examinations were carried out $(\mathrm{n}=13)$ during training sessions and inter-examiner agreement was calculated (kappa). Inter-examiner agreement was moderate with Cohen kappa value 0.574 ( $\mathrm{min}$ 0.293, $\max 0.811$ ).

Dental caries lesions were examined visual-tactilely from five tooth surfaces of all except wisdom teeth after drying them with a three-in-one syringe, using light of the unit, an oral mirror, a ball-point dental probe (WHO). Fibre-optic transillumination (FOTI) was used routinely to transilluminate the tooth surfaces (KaVo, Biberbach, Germany). MM used loops during examinations. Caries status was recorded by a dental nurse manually on a predesigned form. If there were any obstacles hindering visibility on the tooth surface (e.g. swollen gingiva, orthodontic appliances), the information was not recorded. Before the examination, dental plaque was removed with a probe, if necessary.

For estimating depth and activity $(+/-)$ of caries lesions, ICDAS criteria was used [28]. The score 0 represented a sound surface, enamel lesions comprised scores 1-2 and inactive score 3, lesions needing restorative treatment comprised active scores 3 and 4-6 (DT) [28]. Restorations, including materials, and missing teeth were also recorded. Simultaneously with ICDAS scores, caries activity $(+/-)$ was recorded using the following criteria: opaque caries lesions with a whitish/yellowish appearance, a rough/soft texture of the surface after gentle probing and location in a plaque stagnation area were categorized as active lesions (+). Inactive lesions (-) were characterised by a whitish/brown/black shiny and arrested appearance, smooth/hard surface on gentle probing [29].

According to the clinical examination using ICDAS criteria, the numbers of decayed teeth (DT) and tooth surfaces (DS) and decayed, missing, and filled teeth (DMFT) and tooth surfaces (DMFS) were calculated, in order to indicate caries prevalence and severity. The prevalence of initial lesions will be reported elsewhere.

\section{Assessment of anthropometric factors}

Body composition was assessed according to the protocol of the Panic study. Body size and composition were described as body weight $(\mathrm{kg})$, body height $(\mathrm{cm})$, waist circumference $(\mathrm{cm})$, BMI, body mass index standard deviation score (BMI-SDS), and body fat percentage (BF\%). Body weight was measured twice after overnight fasting, bladder emptied, standing upright, and in light underwear using a calibrated InBody ${ }^{\circledR} 720$ bioelectrical impedance device (Biospace, Seoul, South Korea) to an accuracy of $0.1 \mathrm{~kg}$. The mean of these two values was used in the analyses. Body height was measured three times in the Frankfurt plane without shoes by a wall-mounted stadiometer to an accuracy of $0.1 \mathrm{~cm}$. The mean of the two nearest values was used in the analyses. Waist circumference was measured three times at the end of expiration at mid-distance between the bottom of the rib cage and the top of the iliac crest with a non-stretchable measuring tape to an accuracy of $0.1 \mathrm{~cm}$. The mean of the two nearest values was used in the analyses. BMI was calculated as body weight in kilograms divided by body height in meters squared. Participants were categorized to normal weight, overweight and obese according to IOTF criteria [30]. BMI-SDS was computed using the national references [31]. For the analyses, BMI and BMI-SDS were further divided into equally sized tertiles based on their distributions. Mean BMI values in these tertiles ranged: lowest (range 14.9-19.6), middle (range 19.6-21.2) and highest (range 21.2-38.7). The corresponding figures for mean BMI-SDS were: lowest (range -3.0 to -0.4 ), middle (range-0.4-0.2) and highest (range 0.2-2.8). BF\% was measured bladder emptied, in supine position, in light clothing and after removing all metal objects, using a Lunar Prodigy Advance ${ }^{\circledR}$ dual-energy X-ray absorptiometry device (DXA, GE Medical Systems, Madison, Wisconsin, USA) [32].

\section{Assessment of health-related behaviour}

The intake of energy, carbohydrates and sucrose was assessed using food records and data of the Panic study. The food records covered 4 predefined and consecutive days, including 2 weekdays and 2 weekend days or 3 weekdays and 1 weekend day. Clinical nutritionists checked the returned food records together with the adolescent, asked for any missing details e.g. about the quality and brands of the consumed products, recipes and cooking habits of the meals, and portion sizes using picture booklet of portion sizes. They defined main meals and snacks according to the recorded time and type of foods. Breakfast, lunch and dinner were classified as main meals and all eating and drinking occasions between them as snacks. Data of the number of main meals per day $(<3 / 3)$ and the number of snacks per day $(<2 / 2-3 />3)$ were recorded. Nutrient intake was calculated using the Micro Nutrica ${ }^{\circledR}$ dietary analysis software, Version 2.5. This software is based on detailed up-to-date information about the nutrient content of foods in Finland and other countries [33]. Moreover, a clinical nutritionist updated the software by adding new food items and products with their precise nutrient content based on new data in the Finnish food composition database [34] or received from the producers. The frequency of use of SSB (fizzy and energy drinks) was assessed by a questionnaire (never/less than once a week/once a week/2 
times a week/3 times a week/4 times a week/5 times a week/6 times a week/every day).

Tooth brushing habits were queried with following questions: How often do you brush your teeth? (more often than twice a day/twice a day/once a day/many times per week/once a week or more seldom/never); How do you normally brush your teeth? (with a manual toothbrush/with an electric toothbrush/I don't brush my teeth); How often do you usually do interdental cleaning? (more often than twice a day/twice a day/once a day/ many times per week/once a week or more seldom/never). Smoking, electronic cigarette smoking and use of snuff frequency were assessed by the questions: Have you ever smoked/used electronic cigarettes/used snuff? (never/I have done it earlier every now and then but stopped it/ I have done it earlier regularly for $x$ (how many?) years but stopped it/I smoke every now and then but less than once a week/I smoke every now and then $x$ (how many?) times per week/I smoke regularly every day or nearly every day).

\section{Assessment of socioeconomic status (SES)}

Background information was obtained from data of the Panic study and comprised household income (EUR $\leq 29$ 999/30000-59 000/ $\geq 60$ 000) and parental education (vocational/polytechnic/university).

\section{Statistics}

Analyses were made using the SAS software 9.4.8 Copyright (c) 2002-2012 by SAS Institute Inc., Cary, NC, USA) and SPSS version 25 (IBM, New York, USA).

For statistical analyses, the responses from the use of SSB were categorised as follows: once a week $/ 2-3$ times a week/4-7 times a week. The responses to the questions concerning tooth brushing were dichotomized as follows: at least twice a day/less often. For smoking, electronic cigarette smoking and frequency of use of snuff the responses were: never smoked or used/previously or currently smoking or using occasionally or frequently. For analyses, the continuous variables BMI, BMI-SDS and carbohydrate (E\%) and sucrose (E\%) intakes were divided into equally sized tertiles based on their distributions.

Cross-tabulation with chi-square tests was used to analyse the differences between the categorical variables. To compare means, the independent samples T-test was used for variables with normal distributions and the Mann-Whitney-test for variables with skewed distribution. (Tables 1, 2, 3).

Regression models were used to investigate simultaneously associations between caries and anthropometric and behavioural factors. BMI was chosen to indicate anthropometrics, and health-related behaviours (tooth brushing frequency, smoking, use of snuff and diet) based on their contribution to the occurrence of any decayed teeth $(\mathrm{DT}>0)$ in bivariate analyses (Table 4$)$. Associations were further adjusted for the PANIC intervention group, gender, socioeconomics, sucrose intake (E\%) and caries severity at baseline. The occurrence of any decayed teeth $(\mathrm{DT}>0)$ was used as the dependent variable in logistic regression and the number of decayed teeth in Poisson regression. The Odds Ratio (OR) and Incidence Rate Ratio (IRR) values were calculated with $95 \%$ confidence intervals.

For the inter-examiner agreement, the Cohen-kappa value was calculated for dental caries detection between the examiner and the senior researcher on 13 patients. The kappa value was calculated as a mean of all ICDAS scores (0-6) recorded concerning occlusal surfaces in all teeth for all individuals and given here as their mean (min, max).

\section{Results}

The study population comprised 96 girls and 106 boys, with the mean age of 16.5 (SD 0.5) years. Of the participants, $64 \%$ belonged to the intervention group. Boys were on average leaner than girls, their mean BMI being 20.8 (SD 3.3) (Table 1). According to IOTF criteria, $87.3 \%$ of the participants were of normal weight, $8.7 \%$ were overweight and $4.0 \%$ were obese, none were underweight. The total intake of carbohydrates and sucrose in grams was higher for boys than girls, whereas the opposite was true for E\% of carbohydrates and sucrose. Amount of sucrose in $\mathrm{E} \%$ exceeded the recommended $10 \mathrm{E} \%$ in about $50 \%$ of the participants. There was no significant difference between the intervention and control groups $(p=0.450)$. Most of the participants brushed their teeth at least twice daily. Half of the participants used an electric toothbrush and one fifth performed interdental cleaning at least once a day. Electronic cigarette smoking was more common than smoking, specifically among boys $(20 \%)(p<0.001)$. The same was true for use of snuff. Of the boys, $23 \%$ used or had used snuff, whereas the proportion for girls was only $5 \%$. The proportion of daily snuff users was $1 \%$ (Table 1).

Table 2 presents caries indices in the study population. On average, DMFT was 2.4 and DT comprised on average $26 \%$ of the DMFT-values. More than one third (36\%) of the participants had DMFT $=0$ and $74 \%$ were free of dental caries lesions. All caries indices were higher for boys than girls and the same was true for prevalence, yet the differences were not statistically significant between the genders (Table 2).

In bivariate analyses, frequent use of sugar sweetened beverages (SSB) and not having used snuff associated significantly with caries prevalence, whereas higher intake of carbohydrates (E\%), infrequent toothbrushing and number of decayed teeth at baseline associated almost 
Table 1 Characteristics of participants by gender

\begin{tabular}{|c|c|c|c|c|}
\hline & All $(n=202)$ & Girls $(n=96)$ & Boys $(n=106)$ & $P$-value \\
\hline Group (\%) & & & & 0.702 \\
\hline Intervention & 64 & 63 & 65 & \\
\hline Control & 36 & 37 & 35 & \\
\hline Age (years), mean (SD) & $16.5(0.5)$ & $16.4(0.5)$ & $16.5(0.5)$ & 0.378 \\
\hline \multicolumn{5}{|l|}{ Socioeconomics } \\
\hline Parental education ${ }^{1}(\%)$ & & & & 0.028 \\
\hline Vocational & 13 & 12 & 14 & \\
\hline Polytechnic & 41 & 51 & 32 & \\
\hline University & 46 & 37 & 54 & \\
\hline Household income ${ }^{2}(\%)$ & & & & 0.132 \\
\hline$\leq 29,999 €$ & 7 & 5 & 9 & \\
\hline $30,000-59,000 €$ & 23 & 29 & 87 & \\
\hline$\geq 60,000 €$ & 70 & 67 & 73 & \\
\hline \multicolumn{5}{|l|}{ Health-related behaviour } \\
\hline Tooth brushing frequency ${ }^{3}(\%)$ & & & & 0.003 \\
\hline At least twice a day & 73 & 83 & 64 & \\
\hline Less than twice a day & 27 & 17 & 36 & \\
\hline Brushing device ${ }^{3}(\%)$ & & & & 0.332 \\
\hline Electronic toothbrush & 48 & 44 & 52 & \\
\hline Toothbrush & 52 & 56 & 48 & \\
\hline Interdental cleaning frequency ${ }^{3}(\%)$ & & & & 0.533 \\
\hline At least once a day & 21 & 23 & 20 & \\
\hline Less than once a day & 79 & 77 & 80 & \\
\hline Smoking $\left.{ }^{4} \%\right)$ & & & & 0.615 \\
\hline Never smoker & 92 & 93 & 91 & \\
\hline Current of former & 8 & 7 & 9 & \\
\hline Electronic cigarette smoking $\left.{ }^{4} \%\right)$ & & & & $<0.001$ \\
\hline Never smoker & 83 & 95 & 71 & \\
\hline Current or former & 17 & 5 & 29 & \\
\hline Use of snuff ${ }^{4}(\%)$ & & & & $<0.001$ \\
\hline Never & 86 & 95 & 77 & \\
\hline Current or former & 14 & 5 & 23 & \\
\hline Alcohol consumption ${ }^{5}(\%)$ & & & & 0.924 \\
\hline None or quitted & 86 & 86 & 86 & \\
\hline Yes & 14 & 14 & 14 & \\
\hline \multicolumn{5}{|l|}{ Diet } \\
\hline Use of sugar sweetened beverages ${ }^{7}(\%)$ & & & & $<0.001$ \\
\hline Once a week or less often & 68 & 83 & 54 & \\
\hline 2-3 times a week & 22 & 14 & 31 & \\
\hline 4-7 times a week & 10 & 3 & 15 & \\
\hline Number of main meals per day ${ }^{8}(\%)$ & & & & 0.878 \\
\hline$<3$ & 78 & 78 & 79 & \\
\hline 3 & 22 & 22 & 21 & \\
\hline Number of snacks per day ${ }^{8}(\%)$ & & & & 0.253 \\
\hline$<2$ & 43 & 37 & 47 & \\
\hline 2 or 3 & 42 & 44 & 41 & \\
\hline$>3$ & 15 & 19 & 12 & \\
\hline Energy intake (kcal/d), mean (SD) & $1841(511.1)$ & $1655.9(419.6)$ & $2022.7(529.2)$ & $<0.001$ \\
\hline Carbohydrate intake (g/day), mean (SD) & $216.5(66.6)$ & $200.9(55.0)$ & $231.8(73.4)$ & 0.001 \\
\hline
\end{tabular}


Table 1 (continued)

\begin{tabular}{|c|c|c|c|c|}
\hline & All $(n=202)$ & Girls $(n=96)$ & Boys $(n=106)$ & $P$-value \\
\hline Sucrose intake (g/day), mean (SD) & $48.2(25.8)$ & $46.3(23.3)$ & $50.1(28.0)$ & 0.317 \\
\hline Carbohydrate (E\%), mean (SD) & $47.2(7.2)$ & $48.7(6.2)$ & $45.8(7.8)$ & 0.007 \\
\hline Sucrose (E\%), mean (SD) & $10.5(5.1)$ & $11.2(5.1)$ & $9.9(5.2)$ & 0.082 \\
\hline \multicolumn{5}{|l|}{ Anthropometrics } \\
\hline Body height (cm), mean (SD) & $171.2(8.4)$ & $165.7(5.8)$ & $176.1(7.3)$ & $<0.001$ \\
\hline Body weight (kg), mean (SD) & $61.3(11.9)$ & $57.3(8.3)$ & $64.8(13.5)$ & $<0.001$ \\
\hline Waist circumference $(\mathrm{cm})$, mean (SD) & $72.7(8.8)$ & $69.7(6.1)$ & $75.4(10.0)$ & $<0.001$ \\
\hline Body mass index $\left(\mathrm{kg} / \mathrm{m}^{2}\right)$, mean (SD) & $20.8(3.3)$ & $20.9(2.9)$ & $20.8(3.7)$ & 0.962 \\
\hline Body mass index SDS ${ }^{9}$, mean (SD) & $-0.1(1.0)$ & $0.01(0.8)$ & $-0.2(1.1)$ & 0.204 \\
\hline Body fat (\%), mean (SD) & $22.6(9.9)$ & $28.4(6.6)$ & $17.3(9.4)$ & $<0.001$ \\
\hline
\end{tabular}

$P$-values are from independent samples T-test for variables with normal distributions or Mann-Whitney test for variables with skewed distribution and chi-square test for categorical variables. Bolded values indicate statistically significant associations $(P<0.05)$

Missing data: ${ }^{1} \mathrm{n}=16,{ }^{2} \mathrm{n}=20,{ }^{3} \mathrm{n}=15,{ }^{4} \mathrm{n}=2,{ }^{5} \mathrm{n}=1,{ }^{6} \mathrm{n}=15,{ }^{7} \mathrm{n}=1,{ }^{8} \mathrm{n}=16$

${ }^{9}$ standard deviation score

Table 2 Caries severity and prevalence (\%) by gender according to the International Caries Detection and Assessment System $(\mathrm{ICDAS3}+)$

\begin{tabular}{|c|c|c|c|c|}
\hline \multirow[b]{2}{*}{ Number of } & \multirow[t]{2}{*}{ All $(n=202)$} & Girls $(n=96)$ & Boys $(n=106)$ & $P$-value ${ }^{1}$ \\
\hline & & \multicolumn{3}{|l|}{ Mean (SD) } \\
\hline Decayed surfaces & $0.63(1.41)$ & $0.47(0.89)$ & $0.77(1.74)$ & 0.880 \\
\hline Decayed, missed, and filled surfaces & $4.03(5.86)$ & $3.39(4.24)$ & $4.62(6.98)$ & 0.389 \\
\hline Decayed teeth & $0.59(1.27)$ & $0.47(0.89)$ & $0.71(1.52)$ & 0.887 \\
\hline Decayed, missed, and filled teeth & $2.37(2.85)$ & $2.10(2.34)$ & $2.61(3.23)$ & 0.406 \\
\hline \multicolumn{2}{|l|}{ At least one } & \multicolumn{3}{|l|}{ n (\%) } \\
\hline Decayed surface & $53(26)$ & $26(27)$ & $27(25)$ & 0.795 \\
\hline Decayed, missed, or filled surface & $130(64)$ & $58(60)$ & $72(68)$ & 0.266 \\
\hline Decayed tooth & $53(26)$ & $26(27)$ & $27(25)$ & 0.795 \\
\hline Decayed, missed, or filled tooth & $130(64)$ & $58(60)$ & $72(68)$ & 0.266 \\
\hline
\end{tabular}

${ }^{1}$ Mann-Whitney test, ${ }^{1}$ chi-squared test

significantly with DT $>0$. Neither gender nor any anthropometric measures were associated with present caries experience (DT) (Table 3).

In adjusted regression analyses, belonging to the intervention group rather than the control group significantly associated with a lower number of DT. Higher carbohydrate intake (E\%) associated with higher odds of having any decayed teeth $(\mathrm{DT}>0)$ (Table 4$)$. The opposite was true for less frequent use of SSB compared to its daily or almost daily use. In addition, higher carbohydrate intake and tooth brushing frequency less often than twice a day significantly associated with higher number of DT or severity of dental caries. Current or previous use of snuff compared to no history of snuff use as well as less frequent use of SSB compared to daily or almost daily use were significantly associated with a lower number of DT (Table 4).

\section{Discussion}

Caries prevalence in the present study population was low-about $25 \%$ had treatment need. Neither gender nor anthropometric measures or sociodemographic factors were associated with present caries experience (DT). The participants were on average leaner than counterparts in general population. Yet, two thirds consumed sugar sweetened beverages (SSB) weekly. Frequent use of SSB associated significantly with caries prevalence, the same was true for high carbohydrate intake (E\%). High SSB consumption and carbohydrate intake and tooth brushing frequency less often than twice a day significantly associated with higher number of DT or severity of dental caries. On the other hand, receiving dietary counselling (intervention group) significantly associated with a lower number of DT 
Table 3 Distribution of participants according to number of decayed teeth (DT) (ICDAS3+) by intervention group, gender, socioeconomics, oral health-related behaviour and anthropometrics

\begin{tabular}{|c|c|c|c|}
\hline & \multicolumn{3}{|l|}{ DT } \\
\hline & $0(n=149)$ & $\geq 1(n=53)$ & $p$-value \\
\hline Group & & & 0.343 \\
\hline Intervention & 76 & 24 & \\
\hline Control & 70 & 30 & \\
\hline Gender & & & 0.795 \\
\hline Boys & 75 & 25 & \\
\hline Girls & 73 & 27 & \\
\hline \multicolumn{4}{|l|}{ Socioeconomics } \\
\hline Parental education (\%) $)^{1}$ & & & 0.769 \\
\hline Vocational & 75 & 25 & \\
\hline Polytechnic & 73 & 27 & \\
\hline University & 78 & 22 & \\
\hline Household income $(\%)^{2}$ & & & 0.773 \\
\hline$\leq 29,999 €$ & 69 & 31 & \\
\hline $30,000-59,000 €$ & 79 & 21 & \\
\hline$\geq 60,000 €$ & 75 & 25 & \\
\hline \multicolumn{4}{|l|}{ Health-related behavior } \\
\hline Tooth brushing frequency $(\%)^{3}$ & & & 0.066 \\
\hline At least twice a day & 77 & 23 & \\
\hline Less often & 63 & 37 & \\
\hline Smoking $(\%)^{4}$ & & & 0.846 \\
\hline Never & 74 & 26 & \\
\hline Current or occasional & 76 & 24 & \\
\hline Electronic cigarette smoking $(\%)^{4}$ & & & 0.373 \\
\hline Never & 72 & 27 & \\
\hline Current or occasional & 80 & 20 & \\
\hline Use of snuff $(\%)^{4}$ & & & 0.038 \\
\hline Never & 71 & 29 & \\
\hline Current or occasional & 90 & 10 & \\
\hline Smoking or use of snuff (\%) & & & 0.202 \\
\hline Both & 89 & 11 & \\
\hline Smoking & 63 & 38 & \\
\hline Snuff & 90 & 10 & \\
\hline Neither & 72 & 28 & \\
\hline \multicolumn{4}{|l|}{ DIET } \\
\hline Use of sugar sweetened beverages $(\%)^{5}$ & & & 0.033 \\
\hline Once a week or less often & 79 & 21 & \\
\hline 2-3 times a week & 69 & 31 & \\
\hline 4-7 times a week & 53 & 47 & \\
\hline Number of main meals per day $(\%)^{6}$ & & & 0.714 \\
\hline$<3$ & 75 & 25 & \\
\hline 3 & 73 & 28 & \\
\hline Number of snacks per day $(\%)^{6}$ & & & 0.865 \\
\hline$<2$ & 75 & 25 & \\
\hline $2-3$ & 73 & 27 & \\
\hline$>3$ & 79 & 21 & \\
\hline \multicolumn{4}{|l|}{ Carbohydrate intake $(\mathrm{E} \%)^{7}$} \\
\hline Continuous, mean (SD) & $46.6(7.4)$ & $49.0(6.3)$ & 0.057 \\
\hline
\end{tabular}


Table 3 (continued)

\begin{tabular}{|c|c|c|c|}
\hline & \multicolumn{3}{|l|}{ DT } \\
\hline & $0(n=149)$ & $\geq 1(n=53)$ & $p$-value \\
\hline In tertiles (\%) & & & 0.147 \\
\hline Lowest (range 25.7-44.5) & 84 & 16 & \\
\hline Middle (range 44.6-49.7) & 73 & 27 & \\
\hline Highest (range 48.7-66.1) & 69 & 31 & \\
\hline Sucrose intake $(E \%)^{7}$ & $10.3(5.4)$ & $11.1(4.5)$ & 0.185 \\
\hline \multicolumn{4}{|l|}{ Continuous, mean (SD) } \\
\hline In tertiles(\%) & & & 0.381 \\
\hline Lowest (range 1.5-7.8) & 82 & 18 & \\
\hline Middle (range 7.9-11.8) & 73 & 27 & \\
\hline Highest (range 11.9-29.1) & 70 & 30 & \\
\hline \multicolumn{4}{|l|}{ Body composition } \\
\hline Waist (cm), mean (SD) & $72.5(8.2)$ & $73.3(10.4)$ & 0.875 \\
\hline Weight (kg), mean (SD) & $61.2(11.4)$ & $61.5(13.4)$ & 0.911 \\
\hline \multicolumn{4}{|l|}{ Body Mass Index } \\
\hline Continuous, mean (SD) & $20.8(3.1)$ & $21.0(4.0)$ & 0.963 \\
\hline In tertiles (\%) & & & 0.808 \\
\hline Lowest (range 14.9-19.6) & 33 & 34 & \\
\hline Middle (range 19.6-21.2) & 35 & 30 & \\
\hline Highest (range 21.2-38.7) & 32 & 36 & \\
\hline \multicolumn{4}{|l|}{ BMI-SDS $^{8}$} \\
\hline Continuous, mean (SD) & $-0.08(0.9)$ & $-0.08(1.1)$ & 0.874 \\
\hline In tertiles (\%) & & & 0.980 \\
\hline Lowest (range -3.0 to -0.4 ) & 75 & 25 & \\
\hline Middle (range -0.4 to 0.2 ) & 74 & 26 & \\
\hline Highest (range 0.2-2.8) & 73 & 27 & \\
\hline Body fat (\%), mean (SD) & $21.9(9.6)$ & $24.5(10.5)$ & 0.102 \\
\hline \multicolumn{4}{|l|}{ Previous caries experience } \\
\hline \multicolumn{4}{|l|}{ Number of decayed teeth at baseline, mean (SD) } \\
\hline Initial or decayed lesions in deciduous or permanent teeth & $1.1(1.5)$ & $1.7(2.0)$ & 0.052 \\
\hline Only decayed lesions in deciduous or permanent teeth & $0.03(0.2)$ & $0.04(0.3)$ & 0.745 \\
\hline \multicolumn{4}{|l|}{ Number of decayed teeth at 2 year follow-up, mean (SD) } \\
\hline Initial or decayed lesions in deciduous or permanent teeth & $1.2(1.6)$ & $1.7(2.0)$ & 0.095 \\
\hline Only decayed lesions in deciduous or permanent teeth & $0.1(0.5)$ & $0.2(0.5)$ & 0.164 \\
\hline
\end{tabular}

$P$-values are from independent samples T-test for variables with normal distributions (Carbohydrate intake (E\%), Sucrose intake (E\%) and BF (\%)) or Mann-Whitney test for variables with skewed distribution and chi-squared test for categorical variables. Bolded values indicate statistically significant associations $(P<0.05)$

Missing data: ${ }^{1} \mathrm{n}=20,{ }^{2} \mathrm{n}=16,{ }^{3} \mathrm{n}=15^{4} \mathrm{n}=2,{ }^{5} \mathrm{n}=1,{ }^{6} \mathrm{n}=16,{ }^{7} \mathrm{n}=18,{ }^{8}=$ standard deviation score

compared with the controls. Use of snuff appeared to be a protective factor for caries prevalence and severity.

In 2009, DMFT for Finnish 12-year-olds was 0.7, DT 0.3 and the proportion of caries-free $(\mathrm{DMFT}=0)$ was $26 \%$. For 17-year-olds the numbers were $1.3,0.4$ and only 7.3\%, respectively [4]. In 2011 DMFT for Finnish army conscripts (on average almost 20 years), was 4.1, DT 1.4 and $23.1 \%$ were caries-free. According to Finnish national statistics, approximately $60 \%$ of 12 -year-olds had DMFT 0 in 2018 [21]. Offering new data on caries prevalence is a benefit of this study. Indeed, we do not have other current information on the DMFT of Finnish young people of the same age as our participants, but the trend is in line with our findings in all the studies and statistics mentioned above.

An association between childhood anthropometric measurements and dental caries has been widely studied, but the results remain inconclusive $[13,15,35]$. According to Hooley et al. [13], both high and low BMI show association with caries. The association is different depending on the country's developmental stage and socioeconomic situation [13, 14]. Chen et al. [35] 
Table 4 Adjusted logistic regression for occurrence of any decayed teeth (DT > 0) and Poisson regression for number of decayed teeth (DT) (ICDAS3+)

\begin{tabular}{|c|c|c|c|c|}
\hline & \multicolumn{2}{|l|}{$\mathrm{DT}>0(n=150)$} & \multicolumn{2}{|l|}{ DT $(n=150)$} \\
\hline & OR $(95 \% \mathrm{Cl})$ & $p$-value & IRR (95\%CI) & $p$-value \\
\hline \multicolumn{5}{|l|}{ Group } \\
\hline Intervention & Ref & & Ref & \\
\hline Control & $1.7(0.7-3.9)$ & 0.250 & $1.7(1.1-2.8)$ & 0.030 \\
\hline \multicolumn{5}{|l|}{ Gender } \\
\hline Girls & $1.0(0.4-2.6)$ & 0.980 & $1.0(0.6-1.7)$ & 0.963 \\
\hline Boys & Ref & & Ref & \\
\hline \multicolumn{5}{|l|}{ Household income } \\
\hline$\leq 29,999 €$ & Ref & & Ref & \\
\hline $30000-59000 €$ & $1.1(0.2-7.7)$ & 0.925 & $1.0(0.3-2.9)$ & 0.936 \\
\hline$\geq 60,000 €$ & $1.5(0.3-8.7)$ & 0.636 & $1.0(0.4-2.7)$ & 0.993 \\
\hline \multicolumn{5}{|l|}{ Tooth brushing frequency (\%) } \\
\hline At least twice a day & Ref & & Ref & \\
\hline Less often & $1.8(0.6-4.9)$ & 0.264 & $2.2(1.3-3.8)$ & 0.004 \\
\hline \multicolumn{5}{|l|}{ Smoking (\%) } \\
\hline Never & Ref & & Ref & \\
\hline Current or occasional & $0.7(0.1-5.3)$ & 0.770 & $1.0(0.3-2.7)$ & 0.939 \\
\hline \multicolumn{5}{|l|}{ Use of snuff (\%) } \\
\hline Never & Ref & & Ref & \\
\hline Current or occasional & $0.1(0.01-1.0)$ & 0.055 & $0.3(0.1-0.9)$ & 0.028 \\
\hline \multicolumn{5}{|l|}{ Use of sugar sweetened beverages (\%) } \\
\hline Once a week or less often & $0.1(0.01-0.4)$ & 0.006 & $0.1(0.1-0.3)$ & $<0.001$ \\
\hline 2-3 times a week & $0.2(0.02-1.2)$ & 0.070 & $0.3(0.2-0.7)$ & 0.004 \\
\hline 4-7 times a week & Ref & & Ref & \\
\hline Carbohydrate intake (E\%) & $1.1(1.0-1.2)$ & 0.011 & $1.1(1.0-1.1)$ & 0.002 \\
\hline Sucrose intake (E\%) & $0.9(0.9-1.0)$ & 0.260 & $1.0(0.9-1.0)$ & 0.232 \\
\hline Number of decayed teeth at baseline ${ }^{1}$ & $1.1(0.8-1.4)$ & 0.714 & $1.0(0.8-1.2)$ & 0.970 \\
\hline \multicolumn{5}{|l|}{ Body Mass Index tertiles } \\
\hline Lowest (range 14.9-19.6) & $0.8(0.3-2.3)$ & 0.633 & $1.1(0.5-2.1)$ & 0.878 \\
\hline Middle (range 19.6-21.2) & $0.7(0.2-2.1)$ & 0.501 & $0.7(0.4-1.5)$ & 0.431 \\
\hline Highest (range 21.2-8.7) & Ref & & Ref & \\
\hline
\end{tabular}

Bolded values indicate statistically significant associations $(P<0.05)$

${ }^{1}$ Initial or decayed lesions in deciduous or permanent teeth

in their systematic review found that obese children from high-income countries were more likely to have a higher caries prevalence than from low-income countries. However no association between anthropometric measurements and dental caries was discovered in this study. In the PANIC study the population sample comprised predominantly healthy young teenagers. Boys and girls were slimmer compared to other Finnish adolescents in the same age group according to the National School Oral Health Survey [36]. About 13\% of the participants were overweight or obese, whereas the national figure is $17 \%$. This may explain why no association between dental caries and anthropometrics was discovered.
Frequent use of SSB was associated with presence of any decayed teeth $(\mathrm{DT}>0)$ and with severity of dental caries (high DT-value). Despite the participants being part of the PANIC study and about $60 \%$ of them having received information on healthy nutrition 13 times after baseline [24], 60\% of them still used SSB weekly, boys significantly more often than girls. The amount of sucrose in the diet (E\%) exceeded the recommended $10 \mathrm{E} \%$ in about half of the participants. Both the amount of sugars and the frequency of consumption are risk factors for the development of dental caries lesions [37]. Especially added sugar and sugars naturally present in honey, syrups and fruit juices and concentrates are all harmful to teeth [37]. Higher intake of sugars has also been 
associated with obesity [38]. The most consistent association has been between a high intake of SSB and the development of obesity seen also in children [39]. During the past decades more beverages have been consumed than ever before [16]. The findings indicated positive impact of the PANIC intervention on dental caries occurrence and further evaluation would be interesting. PANIC offers data for investigating this and the outcome could benefit for influential education.

Low SES has been associated with higher risk for dental caries in many studies [40]. However, children from high SES groups may also have an increased risk of dental caries, especially in developing countries [14]. In our study most of the participants came from families whose household income as well as parental education was high compared to the general Finnish population [41, 42]. Additionally, most had good health promoting oral and general habits. This must be born in mind while interpreting the present findings and making generalisations to the general population. However, the findings indicate behavioural patterns of teenagers concerning the use of sweet drinks, smoking and as a more recent one, snuffing.

There may be regional differences in caries occurrence. Our sample is from Kuopio City, which is the 9th largest city in Finland, with 118,000 inhabitants and situated in Eastern Finland. Both Widström and Järvinen [4] and Kämppi et al. [43] found that in Southern Finland oral health was better than in the north of the country and that more caries lesions can be detected in small rural towns than in big cities. Manifested dental caries is never a harmless condition; even low levels of dental caries in children and adolescents are of concern, because caries is a lifelong progressive and cumulative disease [44]. In our study, those who had more initial and decayed teeth at baseline also had significantly more caries experience as teenagers. However, when adjusted for behaviour and other covariates, the influence of caries history was no longer significant. The reason for this may be that prevalence of dental caries was very low even at baseline. We do not have comparable data of today's caries situation in different parts of Finland among children and adolescents. Data collected during the 2000s is based on national patient registry data, which is not all-inclusive due to individual recall systems in municipalities.

A significant association was found between irregular tooth brushing and prevalence of dental caries lesions (DT). This still emphasizes the role of regular tooth brushing twice a day with fluoride toothpaste which has been the main reason for declining caries in the past [45]. In our study the majority, $64 \%$ of boys and $83 \%$ of girls, brushed their teeth at least twice a day which is better than on average in this age group in Finland. In the nationwide Finnish School Health Survey of 2019, only
$48 \%$ of $15-16$-year-old boys and $71 \%$ of girls brushed their teeth more than once a day [22]. The results show the need for oral hygiene promotion specifically targeting the boys.

We found that the use of snuff, but not smoking, was significantly associated with decreased odds for restorative treatment need due to dental caries. This is in line with a Swedish report on young adults [46] whereas in older habitual snuff users the association is not found [47]. In our study the proportion of snuff users and smokers was in line with other same-aged Finnish teenagers [48]. Of our study population, $14 \%$ used or had used snuff and $8 \%$ smoked or had smoked. It can be postulated that snuff use is a more accepted way of using nicotine products than smoking, and that snuff users may be aware of the adverse effects of snuff for oral health, which may be compensated by good oral hygiene [49].

The strength of our study is its population-based and longitudinal study design. Comprehensive data on health and health-related behavioural factors, including dietary ones, were available. Data on previous caries experience, at baseline and at the 2-year follow-up could be included in the analyses. In this second follow-up, dental caries lesions were detected carefully by a trained and calibrated senior dentist and in more detail than previously. All five surfaces of all teeth were examined using visualtactile inspection and FOTI. However, it was not possible to take radiographs in our study. About two thirds of the participants had attended dietary counselling sessions after baseline. Due to the long follow-up period, more health-conscious teenagers may have had more predisposition to participate the 8-year follow-up.

Inter-examiner agreement was moderate. This is most likely due to the fact that agreement was calculated covering all ICDAS scores because the prevalence of caries lesions was low. Usually the cut of point sound/decayed is used in investigating inter-examiner agreement. Using that cut-off point $(0 />$ ICDAS $3+)$ would have produced complete agreement (100\%) and was therefore not used.

\section{Conclusion}

This work provides fresh evidence-based data on the prevalence of dental caries among Finnish children and adolescents, the data at present is non-existent. Caries prevalence was low in both girls and boys, which is in line with national statistical figures. The current study suggests that behavioural, but not anthropometric factors are associated with caries experience. Indeed, healthy behaviours were important also in this low caries prevalence population. In general, the boys and girls who participated in our study had good oral and general health habits. Despite the fact that two thirds of the participants had received dietary counselling, 60\% consumed sugar 
sweetened beverages weekly, boys more frequently than girls and sucrose consumption exceeded 10E\% among half of the participants. This emphasizes the role of lifelong regular health promotion.

\section{Acknowledgements \\ Not applicable for this study.}

\section{Authors' contributions}

MM, study design, clinical examination, analyses, preparing the manuscript. SK, preparing data for analysis, preparing the manuscript. ALS, study design, statistical analyses, preparing the manuscript. A-ME, nutrition adviser, preparing the manuscript. JV, preparing data for analysis. TL, PANIC representative preparing the manuscript. $\mathrm{HV}$, statistical analyses. $\mathrm{VA}$, study design, clinical examination, analyses, preparing the manuscript. All authors read and approved the final manuscript.

\section{Funding}

No external funding has been received for this study.

\section{Availability of data and materials}

The datasets used and/or analysed during the current study available from the corresponding author on reasonable request.

\section{Declarations}

\section{Ethics approval and consent to participate}

The study protocol was approved 10.11.2015/295§ by the Research Ethics Committee of the Kuopio University Hospital District of Northern Savo, Finland. All the participants and their parents gave their informed written consent for participation. A renewed written consent was given for participation in the 8-year follow up study. The study protocol followed guidelines of declaration of Helsinki.

\section{Consent for publication}

All authors have given consent for publication.

\section{Competing interests}

None of the authors declare any conflicts of interest.

\section{Author details}

${ }^{1}$ Institute of Dentistry, Faculty of Health Sciences, University of Eastern Finland, P.O. Box 1627, 70211 Kuopio, Finland. ${ }^{2}$ Department of Oral and Maxillofacial Diseases, Kuopio University Hospital, Kuopio, Finland. ${ }^{3}$ Medical Research Center, Oulu University Hospital and University of Oulu, Oulu, Finland. ${ }^{4}$ Institute of Public Health and Clinical Nutrition, School of Medicine, University of Eastern Finland, Kuopio, Finland. ${ }^{5}$ Institute of Biomedicine, School of Medicine, University of Eastern Finland, Kuopio, Finland. ${ }^{6}$ Department of Clinical Physiology and Nuclear Medicine, Kuopio University Hospital, Kuopio, Finland. ${ }^{7}$ Kuopio Research Institute of Exercise Medicine, Kuopio, Finland. ${ }^{8}$ Infrastructure for Population Studies, Faculty of Medicine, University of Oulu, Oulu, Finland. ${ }^{9}$ Research Unit of Oral Health Sciences, University of Oulu, Oulu, Finland.

Received: 6 April 2021 Accepted: 14 June 2021

Published online: 06 July 2021

\section{References}

1. Bernabe E, Marcenes W, Hernandez CR, Bailey J, Abreu LG, Alipour V, et al. Global, regional, and national levels and trends in burden of oral conditions from 1990 to 2017: a systematic analysis for the Global Burden of Disease 2017 Study. J Dent Res. 2020;99(4):362-73. https://doi.org/10. 1177/0022034520908533.

2. Worldometer - World statistics in real time. https://www.worldometers. info. Accessed 24 Feb 2021

3. Suominen-Taipale AL, Widström E, Sund R. Association of examination rates with children's national caries indices in Finland. Open Dent J. 2009;3(1):59-67. https://doi.org/10.2174/1874210600903010059.
4. Widström $\mathrm{E}$, Järvinen S. Caries prevalence and use of dental services in Finnish children and adolescents in 2009. Oral Health Dent Manag J. 2011:10(Issue 4):185-92.

5. Kassebaum NJ, Bernabé E, Dahiya M, Bhandari B, Murray CJL, Marcenes W. Global burden of untreated caries. J Dent Res. 2015;94(5):650-8. https:// doi.org/10.1177/0022034515573272.

6. MeSH -N.Dictionary. http://www.ncbi.nlm.nih.gov/mesh/?term=anthr opometry. Accessed 24 Feb 2021

7. Dattani B. Brook's clinical pediatric endocrinology. New York: Wiley; 2019.

8. Abarca-Gómez L, Abdeen ZA, Hamid ZA, Abu-Rmeileh NM, Acosta-Cazares $B$, Acuin $C$, et al. Worldwide trends in body-mass index, underweight, overweight, and obesity from 1975 to 2016: a pooled analysis of 2416 population-based measurement studies in 128.9 million children, adolescents, and adults. Lancet. 2017;390(10113):2627-42. https://doi.org/10. 1016/S0140-6736(17)32129-3.

9. Rimpelä A, Rainio S, Pere L, Saarni L, Kautiainen S, Kaltiala-Heino $\mathrm{R}$, et al. Suomalaisten nuorten terveys 1977-2003. Finnish Med J. 2004:59(44):4229-35.

10. National Institute of Health Finland (THL) database. Overweight and obesity of children and adolescents. https://thl.fi/fi/tilastot-ja-data/tilas tot-aiheittain/lapset-nuoret-ja-perheet/lasten-ja-nuorten-ylipaino-ja-lihav uus. Accessed Feb 24th 2021

11. Chi DL, Luu M, Chu F. A scoping review of epidemiologic risk factors for pediatric obesity: implications for future childhood obesity and dental caries prevention research. J Public Health Dent. 2017;77(S1):S8-31. https://doi.org/10.1111/jphd.12221.

12. Cinar AB, Christensen LB, Hede B. Clustering of obesity and dental caries with lifestyle factors among Danish adolescents. Oral Health Prev Dent. 2011;9(2):123.

13. Hooley M, Skouteris H, Boganin C, Satur J, Kilpatrick N. Body mass index and dental caries in children and adolescents: a systematic review of literature published 2004 to 2011. Syst Rev. 2012;1(1):57. https://doi.org/ 10.1186/2046-4053-1-57.

14. Karki S, Päkkilä J, Ryhänen T, Laitala M, Humagain M, Ojaniemi M, et al. Body mass index and dental caries experience in Nepalese schoolchildren. Commun Dent Oral Epidem. 2019;47(4):346-57. https://doi.org/10. $1111 /$ cdoe. 12465 .

15. Li L, Wong HM, Peng S, Mcgrath CP. Anthropometric measurements and dental caries in children: a systematic review of longitudinal studies 123. Adv Nutr. 2015;6(1):52-63. https://doi.org/10.3945/an.114.006395.

16. Popkin BM, Adair LS, Ng SW. Now and then: the global nutrition transition: the pandemic of obesity in developing countries. Nutr Rev. 2012;70(1):3-21. https://doi.org/10.1111/j.1753-4887.2011.00456.x.

17. Juul F, Hemmingsson E. Trends in consumption of ultra-processed foods and obesity in Sweden between 1960 and 2010. Public Health Nutr. 2015:18(17):3096-107. https://doi.org/10.1017/\$1368980015000506.

18. Kumar S, Kroon J, Lalloo R, Kulkarni S, Johnson NW. Relationship between body mass index and dental caries in children, and the influence of socio-economic status. Int Dent J. 2017;67(2):91-7. https://doi.org/10. 1111/idj.12259.

19. Evans EW, Hayes C, Palmer CA, Bermudez Ol, Cohen SA, Must A. Dietary intake and severe early childhood caries in low-income. Young Children J Acad Nutr Diet. 2013;113(8):1057-61. https://doi.org/10.1016/j.jand.2013. 03.014.

20. Tanner T, Harju L, Päkkilä J, Patinen P, Tjäderhane L, Anttonen V. Consumption of snacks and dental caries among Finnish young men: a crosssectional epidemiological study. Odontol. 2020;108(3):486-92. https:// doi.org/10.1007/s10266-019-00473-z.

21. Statistical information on welfare and health in Finland( Sotkanet).Dental caries prevalence among 12-year-olds. https://sotkanet.fi/sotkanet/fi/ taulukko/?indicator=szZMtYZPt9bNi6-y $1 \mathrm{kOCOVmGAA}==$ \&region= s07MBAA $=\&$ year $=$ sy6rsjbS0zUEAA $==\&$ gender $=m ; f ; t \& a b s=f \& c o l o r=$ f\&buildVersion $=3.0-$ SNAPSHOT\&buildTimestamp $=201911131146$. Accessed Feb 24th 2021

22. Sampo statistics, National Institute of Health Finland. Tooth brushing in School Oral Health Survey. https://sampo.thl.fi/pivot/prod/fi/ktk/ktk1/ summary_perustulokset2?alue_0 $=87869 \&$ mittarit_0 $=187209 \&$ mitta rit_1=200280\&mittarit_2=199373\&vuosi_0=v2017\&kouluaste_0= 161293\#. Accessed Feb 24th 2021 
23. Hoppu U, Lehtisalo J, Tapanainen H, Pietinen P. Dietary habits and nutrient intake of Finnish adolescents. Public Health Nutr. 2010;13(6A):965-72. https://doi.org/10.1017/S1368980010001175.

24. The Physical Activity and Nutrition in Children (PANIC) study, University of Eastern Finland, Kuopio, Finland. Register on the physical activity and nutrition in children (panic) study. https://uefconnect.uef.fi/en/group/ the-physical-activity-and-nutrition-in-children-panic-study/. Accessed Jun 26th 2020

25. Hausen H, Kärkkäinen S, Seppä L. Caries data collected from public health records compared with data based on examinations by trained examiners. Caries Res. 2001;35(5):360-5. https://doi.org/10.1159/000047475.

26. Tanner T, Kämppi A, Päkkilä J, Patinen P, Rosberg J, Karjalainen K, et al. Prevalence and polarization of dental caries among young, healthy adults: cross-sectional epidemiological study. Acta Odontol Scand. 2013;71(6):1436-42. https://doi.org/10.3109/00016357.2013.767932.

27. Karki S, Laitala M, Humagain M, Seppänen M, Päkkila J, Anttonen V. Oral health status associated with sociodemographic factors of Nepalese schoolchildren: a population-based study. Int Dent J. 2018;68(5):348-58. https://doi.org/10.1111/idj.12393.

28. Ismail Al, Sohn W, Tellez M, Amaya A, Sen A, Hasson H, et al. The International Caries Detection and Assessment System (ICDAS): an integrated system for measuring dental caries. Commun Dent Oral Epidemiol. 2007;35(3):170-8. https://doi.org/10.1111/j.1600-0528.2007.00347.x.

29. Nyvad B, Baelum V. Nyvad criteria for caries lesion activity and severity assessment: a validated approach for clinical management and research. Caries Res. 2018;52(5):397-405. https://doi.org/10.1159/000480522.

30. Cole TJ, Lobstein T. Extended international (IOTF) body mass index cutoffs for thinness, overweight and obesity. Pediatr Obes. 2012;7(4):284-94. https://doi.org/10.1111/j.2047-6310.2012.00064.x.

31. Saari A, Sankilampi U, Hannila M, Kiviniemi V, Kesseli K, Dunkel L. New Finnish growth references for children and adolescents aged 0 to 20 years: length/height-for-age, weight-for-length/height, and body mass index-for-age. Ann Med. 2011;43(3):235-48. https://doi.org/10.3109/ 07853890.2010 .515603$.

32. Veijalainen A, Haapala E, Väistö J, Leppänen M, Lintu N, Tompuri T, et al. Associations of physical activity, sedentary time, and cardiorespiratory fitness with heart rate variability in 6- to 9-year-old children: the PANIC study. Eur J Appl Physiol. 2019;119(11):2487-98. https://doi.org/10.1007/ s00421-019-04231-5.

33. Rastas M, Seppänen $R$, Knuts LR, Hakala P, Karttila V. Nutrient composition of foods. The Social Insurance Institution of Finland; 1997.

34. National Food Composition Database in Finland (Fineli). National Institute for Health and Welfare, Nutrition Unit.. Finnish food composition database. Release 19. Helsinki 2018. https://www.google.com/search?client= firefox-b-d\&q=www.fineli.fi. Accessed Feb 24th 2021

35. Chen D, Zhi Q, Zhou Y, Tao Y, Wu L, Lin H. Association between dental caries and bmi in children: a systematic review and meta-analysis. Caries Res. 2018;52(3):230-45. https://doi.org/10.1159/000484988.

36. Sampo statistics, National Institute of Health Finland. Overweight in School Oral Health Survey. https://sampo.thl.fi/pivot/prod/fi/ktk/ktk1/ fact_ktk_ktk1?row $=199385$ L\&column=area-87869.\&column=time403056.\&column $=161293 \mathrm{~L} \&$ column $=$ gender $-143993 \& \mathrm{fo}=1$. Accessed Feb 24th 2021

37. Moynihan P. Sugars and dental caries: evidence for setting a recommended threshold for intake. Adv Nutr (Bethesda, Md). 2016;7(1):149-56. https://doi.org/10.3945/an.115.009365.

38. Te Morenga L, Mallard S, Mann J. Dietary sugars and body weight: systematic review and meta-analyses of randomised controlled trials and cohort studies. BMJ Br Med J. 2013;346(7891):12. https://doi.org/10.1136/ bmj.e7492.

39. Scharf RJ, DeBoer MD. Sugar-sweetened beverages and Children's Health. Annu Rev Pub Health. 2016;37(1):273-93. https://doi.org/10.1146/annur ev-publhealth-032315-021528.

40. Foley M, Akers H. Does poverty cause dental caries? Austr Dent J. 2019;64(1):96-102. https://doi.org/10.1111/adj.12666.

41. Statistics Finland. Educational structure of population updated 2021. http://www.stat.fi/tup/suoluk/suoluk_koulutus_en.html. Accessed Feb 24th 2021

42. Statistics Finland. Income distribution statistics. http://www.stat.fi/til/tit/ 2018/06/tjt_2018_06_2020-03-12_tie_001_en.html. Accessed Feb 24th 2021

43. Kämppi $A$, Tanner T, Päkkilä J, Patinen $P$, Järvelin $M$, Tjäderhane $L$, et al. Geographical distribution of dental caries prevalence and associated factors in young adults in Finland. Caries Res. 2013;47(4):346-54. https://doi. org/10.1159/000346435.

44. Broadbent JM, Thomson WM, Poulton R. Trajectory patterns of dental caries experience in the permanent dentition to the fourth decade of life. J Dent Res. 2008;87(1):69-72. https://doi.org/10.1177/154405910808700 112.

45. Lagerweij MD, van Loveren C. Declining caries trends: are we satisfied? Curr Oral Health Rep. 2015;2(4):212-7. https://doi.org/10.1007/ s40496-015-0064-9.

46. Petersson $\mathrm{GH}$, Twetman S. Tobacco use and caries increment in young adults: a prospective observational study. BMC Res Notes. 2019;12(1):218. https://doi.org/10.1186/s13104-019-4253-9.

47. Hellqvist L, Rolandsson M, Hugoson A, Lingström P, Birkhed D. Dental caries and associated factors in a group of Swedish snus users. Swed Dent J. 2015;39(1):47-54.

48. National Institute of Health Finland (THL)database. Alcohol, tobacco and addictions. https://thl.fi/en/web/alcohol-tobacco-and-addictions. Accessed Feb 24th 2021

49. Päkkilä J, Anttonen V, Patinen P, Nyman K, Valkeapää K, Birkhed D, et al. Profiling of smokers and snuffers among young Finnish men-crosssectional epidemiological study. Acta Odontol Scand. 2017;75(8):577-83. https://doi.org/10.1080/00016357.2017.1361548.

\section{Publisher's Note}

Springer Nature remains neutral with regard to jurisdictional claims in published maps and institutional affiliations.

\footnotetext{
Ready to submit your research? Choose BMC and benefit from:

- fast, convenient online submission

- thorough peer review by experienced researchers in your field

- rapid publication on acceptance

- support for research data, including large and complex data types

- gold Open Access which fosters wider collaboration and increased citations

- maximum visibility for your research: over $100 \mathrm{M}$ website views per year
}

At BMC, research is always in progress.

Learn more biomedcentral.com/submissions 